\title{
LA CLINICA DEI DISTURBI DEL COMPORTAMENTO ALIMENTARE: COMPLICANZE MEDICHE
}

\author{
MASSIMO SCACCHI $(*)$
}

SuNTO. - L'anoressia nervosa è una patologia psichiatrica caratterizzata dall'insorgenza tipicamente in età puberale. Prevalente nel sesso femminile e nelle società industrializzate, è gravata dalla più alta mortalità fra le malattie psichiatriche: a ciò contribuiscono le complicanze organiche del grave stato di denutrizione e l'elevata frequenza di suicidi. La cute delle pazienti è secca, desquamante, pallida-giallognola, coperta di lanugo; fanno inoltre parte del quadro clinico ipotrofia muscolare, disidratazione, disturbi elettrolitici, rallentato svuotamento gastrico, stipsi, disfunzione epatica, leucopenia, bradicardia, ipotensione, prolasso mitralico, versamento pericardico, atrofia corticale cerebrale. Sul versante endocrino, sono descritte anomalie più o meno rilevanti a carico di tutti gli assi funzionali. Tali alterazioni, reversibili con il recupero ponderale o la guarigione, costituiscono epifenomeni della malattia di base. L'ipogonadismo ipogonadotropo, di origine ipotalamica, dà luogo nei soggetti di sesso femminile all'amenorrea, che ha costituito tradizionalmente per lungo tempo uno dei criteri diagnostici della malattia. La secrezione delle gonadotropine riacquista caratteristiche tipiche della fase puberale, mentre appaiono ridotte le concentrazioni sieriche degli steroidi gonadici. La low $\mathrm{T}_{3}$ syndrome, comune ad altri stati di defedamento, deriva da una conversione preferenziale di $T_{4}$ a reverse $T_{3}$ piuttosto che a $T_{3}$ nei tessuti periferici. Questo meccanismo, finalizzato al risparmio energetico, pur contribuendo alla bradicardia e all'intolleranza al freddo delle pazienti anoressiche, non necessita di terapia sostitutiva. L'asse ipotalamo-ipofisi-surrene risulta attivato, come in altre patologie psichiatriche (ad esempio, la depressione): l'ipercortisolismo potrebbe contribuire ad alcune manifestazioni cliniche dell'anoressia nervosa, quali l'osteopenia e l'atrofia corticale cerebrale. Le anomalie dell'asse somatotropo configurano un quadro di resistenza all'azione del GH: a fronte infatti di un'esaltata secrezione somatotropinica, i livelli circolanti di IGF-I risultano francamente ridotti a causa della malnutrizione cronica. Insieme alla carenza di sostanze nutritive e al basso peso corporeo, ipogonadismo, ipercortisolismo e deficit somatomedinico contribuiscono allo sviluppo di una demineralizzazione scheletrica di entità tale da aumentare il rischio di fratture nel lungo termine.

(*) Dipartimento di Scienze Cliniche e di Comunità, Università degli Studi di Milano; Unità Operativa di Medicina Generale ad indirizzo Endocrino-Metabolico, Ospedale San Giuseppe IRCCS, Istituto Auxologico Italiano, Oggebbio-Piancavallo (VB), Italia. E-mail: massimo.scacchi@unimi.it 
$* * *$

ABSTRACT. - Anorexia nervosa, commonly occurring in peripubertal girls, is burdened by the highest mortality rate of any psychiatric disorder, due to the organic complications induced by the severe starvation and the high rate of suicide. Patients display dry, pale and yellowish skin, with lanugo hair growth. The clinical picture is characterized by muscle hypotrophy, dehydration, electrolyte disturbances, delayed gastric emptying, constipation, elevation of liver transaminases, leukopenia, bradycardia, hypotension, mitral valve prolapse, pericardial effusion, brain cortical atrophy. Endocrine complications, reversible with weight gain and recovery, can be interpreted as epiphenomena of the disease. Hypogonadotropic hypogonadism, of hypothalamic origin, is the cause of amenorrhea, which was considered in the past one of the diagnostic criteria of the disease. Gonadotropin secretion is characterized by a prepubertal pattern and serum sex steroid levels are low. The preferential conversion of $T_{4}$ to reverse $T_{3}$ rather than to $T_{3}$ in peripheral tissues, common in all malnourishment states, leads to the so called low $\mathrm{T}_{3}$ syndrome. This mechanism, aimed at saving energy, although contributing to bradycardia in these patients, does not require replacement therapy. Like in other psychiatric disorders such as depression, the hypothalamic-pituitary-adrenal axis is activated: hypercortisolism might contribute to some clinical features of the disease such as osteopenia and brain cortical atrophy. The abnormalities of the somatotropin-somatomedin axis are substantially represented by a picture of acquired GH resistance: indeed, due to chronic malnutrition, circulating IGF-I is frankly low in spite of markedly enhanced GH release. Together with malnourishment and low body weight, hypogonadism, hypercortisolism and IGF-I deficiency contribute to bone demineralization, which in its turn leads to an increased fracture risk in the future of these patients.

L'anoressia nervosa (AN) è una patologia psichiatrica caratterizzata da marcata riduzione ponderale, distorta percezione dell'immagine corporea e aberrazione del comportamento alimentare, finalizzata al mantenimento o all'incremento dello stato di magrezza. L'AN insorge tipicamente in età adolescenziale e predilige il sesso femminile; prevale inoltre nelle società industrializzate, nelle famiglie di ceto medio-elevato e nella razza caucasica. Tuttavia, negli ultimi anni si è assistito ad un progressivo aumento di incidenza nei maschi e ad una maggior diffusione nelle classi socio-economiche meno elevate. E' gravata dalla più alta mortalità fra le malattie psichiatriche: a ciò contribuiscono le complicanze organiche del grave stato di denutrizione e l'elevata frequenza di suicidi. L'85\% dei pazienti rientra in una fascia di età compresa tra 13 e 20 anni, periodo critico per la crescita, lo sviluppo puberale e l'acquisizione di un adeguato picco di massa ossea. Nella bulimia nervosa (BN) le complicanze internistiche sono prevalentemente conseguenti alle condotte di eliminazione, vale a dire autoinduzione del vomito e abuso di lassativi. 


\section{QuAdro CLINICO EXTRAENDOCRINO}

Le complicanze extraendocrine dell'AN, che interessano svariati apparati e sistemi (tegumentario, muscoloscheletrico, gastroenterico, cardiovascolare, emopoietico e nervoso) [1], riconoscono prevalentemente le loro cause da un lato nella restrizione alimentare (con conseguente catabolismo proteico e lipidico e perdita di volume e funzione cellulari) e dall'altro nella messa in atto di condotte di eliminazione (abuso di lassativi e/o diuretici, autoinduzione del vomito, iperattività fisica). Le pazienti sono generalmente disidratate, sia per la restrizione di liquidi che per l'abuso di diuretici; alla disidratazione contribuiscono inoltre una carente risposta dell'adiuretina agli stimoli osmotici ed una ridotta sensibilità del rene all'adiuretina stessa. Gli squilibri elettrolitici, segnatamente l'ipopotassiemia delle pazienti che vomitano e abusano di diuretici, possono essere talmente gravi da essere fatali. L'ipotrofia muscolare è importante. La cute delle pazienti è secca, desquamante pallida-giallognola, coperta di lanugo; comune è l'acrocianosi. Numerosi e frequenti sono i sintomi lamentati a carico dell'apparato digerente: dispepsia, epigastralgie, precoce senso di ripienezza, rigurgito, addominalgie, stipsi ostinata. Nelle pazienti che vomitano frequentemente le ghiandole salivari sono spesso ipertrofiche; inoltre, non sono rare le esofagiti. Lo stomaco è di volume ridotto e la sua muscolatura è ipotonica: ciò determina rallentato svuotamento gastrico e distensione post-prandiale. Sono state inoltre descritte atonia e dilatazione intestinali. Nelle forme severe di AN è possibile osservare un marcato rialzo delle transaminasi, a testimonianza di un serio danno a carico del fegato; in questi casi, una renutrizione eccessiva o affrettata può ulteriormente peggiorare la funzione epatica sino all'insufficienza fatale. Fra le più comuni manifestazioni a carico dell'apparato cardiovascolare sono sicuramente bradicardia e ipotensione. Si segnalano inoltre riduzione della massa ventricolare sinistra e della gittata cardiaca, così come ridotte dimensioni telediastoliche e sistoliche del ventricolo sinistro (verosimilmente legate all'ipovolemia di lunga durata), prolasso della mitrale, versamento pericardico e prolungamento dell'intervallo QT. Anemia e leucopenia sono riscontrabili in almeno un terzo delle pazienti, mentre più rara è la piastrinopenia, che può essere però marcata al punto da determinare porpora. Il midollo è ipoplasico, andando incontro nei casi più seri a degenerazione gelatinosa. Non sembra peraltro presente nell'AN una maggiore suscettibilità alle infezioni. Valutazioni neurora- 
diologiche hanno dimostrato nelle pazienti più gravi una riduzione dello spessore della corteccia cerebrale talvolta sovrapponibile a quanto osservato nella malattia di Alzheimer; va segnalato che il recupero ponderale sembra non associarsi in tempi brevi ad una normalizzazione del quadro neuroradiologico. Nella BN [2] le abbuffate e la frequente autoinduzione del vomito determinano erosioni dentali, carie, gengiviti, ipertrofia parotidea, laringofaringiti e malattia gastroesofagea da reflusso, raramente rottura esofagea (sindrome di Boerhaave). Gli effetti dell'abuso di lassativi sull'apparato gastroenterico sono potenzialmente seri: l'evenienza più temibile è rappresentata dal cosiddetto colon catartico, caratterizzato da perdita della peristalsi, dilatazione e atonia del grosso intestino. Anche nelle paziente bulimiche l'ipopotassiemia può essere estremamente grave.

\section{COMPLICANZE ENDOCRINE}

La maggior parte delle alterazioni endocrine conseguenti all'AN, coinvolgenti gran parte degli assi funzionali, risulta comune ad altri stati di malnutrizione ed è reversibile con la normalizzazione del peso corporeo. Tuttavia, alcune di queste anomalie, segnatamente l'ipogonadismo responsabile dell'amenorrea, possono precedere il calo ponderale e/o persistere dopo normalizzazione del peso: tale riscontro suggerisce la possibile esistenza di disfunzioni primarie della neuroregolazione ipotalamica [3]. La leptina è un ormone proteico prodotto prevalentemente dal tessuto adiposo bianco, ma anche da altri tessuti fra cui alcune linee cellulari ipofisarie (somatotropi, corticotropi, tireotropi e gonadotropi). Tale peptide esercita un ruolo fondamentale nel controllo del peso corporeo, dal momento che riduce l'introito calorico e aumenta la spesa energetica a riposo. I livelli circolanti di leptina sono positivamente correlati con l'estensione delle riserve adipose, e risultano pertanto elevati in malattie caratterizzate da accumulo di grasso corporeo quali l'obesità essenziale, la sindrome di Cushing e il deficit di ormone della crescita $(\mathrm{GH})$ in età adulta. Non stupisce dunque che la leptinemia sia francamente ridotta in una patologia contraddistinta da magrezza estrema quale l'AN. Il recupero ponderale si accompagna ad un aumento della leptina circolante. Le pazienti che presentano, al termine di un programma di recupero ponderale, una elevata leptinemia corrono un rischio maggiore di recidiva di calo ponderale entro l'anno successivo. La lepti- 
na, espressa - così come i suoi recettori - a livello ipofisario, influenza a sua volta la funzione anteroipofisaria. Se è ben documentato il suo effetto stimolante la secrezione gonadotropinica (suggestiva è l'ipotesi di un possibile ruolo dell'ipoleptinemia nella genesi dell'ipogonadismo ipogonadotropo dell'AN), meno chiara è l'influenza esercitata dalla leptina sulla secrezione di GH, fortemente aumentata nell'AN: tale effetto sembra infatti essere stimolatorio nel roditore e inibitorio nell'uomo. Interessanti, ma ancora da verificare, le ipotesi che l'ipoleptinemia possa contribuire all'ipersomatotropinismo e all'attivazione dell'asse ipotalamo-ipofisi-surrene (hypothalamic-pituitary-adrenal, HPA) dell'AN. La ghrelina è un peptide acilato prodotto prevalentemente dalle ghiandole ossintiche dello stomaco, ma anche da altri tessuti tra i quali ipotalamo e ipofisi. Tale ormone, secreto in condizioni di digiuno, promuove l'assunzione di cibo (il suo effetto oressante è stato dimostrato anche nell'uomo dopo somministrazione periferica) e riduce il metabolismo basale. Nell'AN è ben documentata una iperghrelinemia, con livelli circolanti inversamente correlati con il BMI. Lipersecrezione di tale ormone oressante, che molto probabilmente riflette un fisiologico tentativo di compenso nei confronti della mancata assunzione calorica e della carenza di depositi energetici, viene corretta dal recupero ponderale. Sul versante endocrino, la ghrelina, ligando endogeno dei recettori per i GHsecretagoghi (GHS) sintetici, rappresenta uno stimolo fisiologico alla secrezione di GH, prolattina e corticotropina (ACTH). Intrigante, ma ancora dibattuta, l'ipotesi che l'iperghrelinemia possa contribuire all'ipersomatotropinismo e all'ipogonadismo dell'AN. In tale patologia psichiatrica è ben documentata un'iperattività dell'asse HPA, che appare caratterizzato da livelli sierici e urinari di cortisolo ai limiti superiori della norma o francamente elevati, nonché, in circa una metà delle pazienti, da abolizione del ritmo circadiano del cortisolo e della sopprimibilità della cortisolemia da parte di basse dosi di desametasone. L'ipercortisolismo, accompagnato da normali valori di ACTH, è secondario sia ad un ridotto catabolismo del cortisolo, tipico di tutti gli stati di malnutrizione, che ad una sua aumentata produzione. L'attivazione dell'asse è sostenuta da una iperincrezione ipotalamica di corticotropin releasing hormone $(\mathrm{CRH})$, testimoniata indirettamente dalla risposta ridotta di ACTH e cortisolo allo stimolo con $\mathrm{CRH}$ esogeno e direttamente dal riscontro di elevati livelli liquorali del neuroormone in pazienti anoressiche. Assai intrigante è l'osservazione che nell'animale da esperimento la somministrazione intracerebroventricolare di $\mathrm{CRH}$ induce 
aumento dell'attività motoria, riduzione del consumo di alimenti, rallentamento dello svuotamento gastrico e inibizione della secrezione di gonadotropin releasing hormone $(\mathrm{GnRH})$, situazioni tutte presenti nell'AN. L'assenza delle tipiche manifestazioni cliniche dell'ipercortisolismo nell'AN non sembra derivare da una ridotta sensibilità ai glucocorticoidi, ma piuttosto dalla carenza di substrati metabolici necessari per sviluppare l'azione ormonale. Non si può comunque escludere che l'ipercortisolismo giochi un ruolo nella patogenesi dell'osteoporosi, dell'atrofia cerebrale e dell'ipotrofia muscolare di frequente riscontro nell'AN. Per quanto riguarda i rapporti fra leptina e asse HPA, è noto come l'ormone adipocitario sia in grado di modulare in senso negativo l'attività di tale asse nei roditori, verosimilmente attraverso l'inibizione del rilascio di neuropeoptide Y (NPY), peptide in grado di stimolare la secrezione ipotalamica di CRH. Considerati i bassi livelli circolanti di leptina e l'aumento dei livelli liquorali di NPY dell'AN, è ipotizzabile che l'ipoleptinemia possa contribuire all'attivazione dell'asse HPA. Come anticipato, l'amenorrea è stato in passato considerato uno dei criteri diagnostici dell'AN. Nonostante rappresenti una appropriata reazione di adattamento al dimagrimento, la scomparsa dei flussi mestruali precede il calo ponderale in circa la metà delle pazienti e può persistere nonostante il recupero del peso corporeo. La normalizzazione del peso sembra quindi costituire una condizione necessaria ma non sufficiente per la piena correzione della funzione gonadica, che verrebbe ottenuta solo quando anche le condizioni psichiche sono migliorate. L'amenorrea nella donna e il deficit erettile nell'uomo sono determinate da un ipogonadismo ipogonadotropo con bassi livelli plasmatici di gonadotropine (FSH e LH), i cui picchi secretori spontanei sono ridotti o limitati alle ore notturne come nei soggetti prepuberi. Nelle donne anche la risposta delle gonadotropine allo stimolo con $\mathrm{GnRH}$ è sovrapponibile a quella tipica della fase prepuberale, in cui l'aumento di FSH è maggiore e anticipato rispetto a quello di $\mathrm{LH}$; la risposta di $\mathrm{LH}$ al proprio releasing hormone è tanto più compromessa quanto maggiore è il calo ponderale. $\mathrm{Al}$ deficit di gonadotropine consegue la diminuzione dei livelli plasmatici di estradiolo e progesterone. Nel maschio risultano ridotte le concentrazioni sieriche di testosterone e la risposta delle gonadotropine al GnRH. In entrambi i sessi sono aumentati i livelli di sex hormone binding globulin (SHBG), che può essere considerata un attendibile indice nutrizionale: la sua produzione è infatti modulata negativamente dall'insulina, ridotta in condizioni di malnutrizione. Dal punto di vista fisiopatologico, la 
compromissione della secrezione gonadotropinica di questi pazienti è dovuta alla soppressione dell'attività pulsatile dei neuroni ipotalamici secernenti GnRH. In effetti, è stato dimostrato che la leptina stimola fisiologicamente la liberazione di $\mathrm{GnRH}$ dall'ipotalamo e di $\mathrm{LH}$ dall'ipofisi con meccanismo mediato dall'ossido nitrico (NO). L'ipoleptinemia dell'AN determinerebbe, accanto ad una riduzione della sintesi di NO, un aumento della increzione ipotalamica di NPY, sostanza in grado di inibire la secrezione gonadotropinica verosimilmente proprio sopprimendo la liberazione episodica di GnRH. Il trattamento con leptina ricombinante umana si è dimostrato recentemente in grado di ripristinare la secrezione pulsatile di LH e la funzione gonadica in donne con amenorrea ipotalamica causata da esercizio fisico strenuo o basso peso corporeo. Anche la già citata iperincrezione di CRH può contribuire all'ipogonadismo dell'AN; tale peptide è infatti in grado di inibire la secrezione ipotalamica di $\mathrm{GnRH}$, verosimilmente favorendo la secrezione di oppioidi endogeni. Nelle condizioni di digiuno prolungato la T4 viene preferibilmente convertita nei tessuti periferici in reverse T3 (rT3), metabolita biologicamente inattivo, anziché in T3. Questo fenomeno, finalizzato al risparmio energetico, dà luogo alla cosiddetta "low T3 syndrome", in cui si osservano bassi livelli circolanti di T3, livelli normali o solo lievemente ridotti di T4 e normali valori di tireotropina (TSH). Lipotiroidismo compensatorio, pur essendo ritenuto concausa di alcuni sintomi e segni della malattia (stipsi, intolleranza al freddo, bradicardia, ipercarotenemia e ipercolesterolemia), non necessita di terapia sostitutiva e va incontro a correzione spontanea dopo recupero ponderale. L'analisi della secrezione somatotropinica spontanea mediante l'algoritmo computerizzato Cluster dimostra nell'AN un aumento della frequenza degli episodi secretori (con conseguente incremento della componente pulsatile di tale secrezione) ma anche dei livelli ormonali tra $\mathrm{i}$ picchi [4]. Quest'ultimo rilievo non è ascrivibile ad una riduzione della clearance metabolica del $\mathrm{GH}$, ma ad un reale aumento della secrezione basale dell'ormone, come dimostra l'analisi di deconvoluzione dei profili secretori. La responsività del GH ai diversi stimoli sovraipofisari è variabile in queste pazienti. In particolare, le risposte somatropiniche all'ipoglicemia insulinica, alla levo-dopa e alla somministrazione acuta di desametasone sono significativamente compromesse. Al contrario, appaiono nella norma le risposte evocate dall'agonista $\alpha 2$-adrenergico clonidina e dall'aminoacido arginina. Si segnala inoltre nell'AN un aumento paradosso del GH dopo infusione endovenosa di glucosio, che nel soggetto 
normale sopprime invece la secrezione dell'ormone. La risposta del GH alla stimolazione ipofisaria diretta con GHRH risulta normale o addirittura esagerata [5]. Per quanto riguarda il compartimento periferico dell'asse, a fronte di elevati livelli circolanti di GH, le concentrazioni sieriche di insulin-like growth factor-I (IGF-I) appaiono francamente ridotte, così come quelle di IGF binding protein 3 (IGFBP-3), la cui produzione è fisiologicamente stimolata da GH e IGF-I. Va in questo contesto ricordato come, nell'uomo, sia il digiuno che la malnutrizione cronica determinino una marcata compromissione della produzione di somatomedina. Costantemente elevate risultano nell'AN IGFBP-1 e IGFBP-2, la cui sintesi è modulata negativamente dall'insulina, in accordo con la costante ipoinsulinemia di queste pazienti. Da un punto di vista clinico, il deficit di IGF-I può essere in parte responsabile del difetto di crescita staturale osservato nelle pazienti in cui l'AN sia insorta in età precoce. Insieme al deficit nutrizionale e ad altre alterazioni endocrine (ipogonadismo, ipercortisolismo), la carenza di IGF-I può contribuire anche all'osteopenia di queste pazienti. La persistenza di basso IGFI nonostante l'elevato GH suggerisce che l'AN sia una condizione di resistenza acquisita al $\mathrm{GH}$, come altre forme di malnutrizione e gli stati ipercatabolici. Tale ipotesi è rafforzata dal riscontro, sempre nell'AN, di ridotte concentrazioni di growth hormone binding protein (GHBP), corrispondente al dominio extracellulare del recettore del $\mathrm{GH}$, così come della mancata risposta somatomedinica alla somministrazione di GH biosintetico. Oltre la metà delle pazienti affette da AN presenta osteopenia al momento della diagnosi [6]. Tale complicanza arriva ad interessare il 90\% delle pazienti nel corso della malattia. Nel 40\% dei casi è dimostrabile vera e propria osteoporosi. Come già osservato, l'insorgenza dell'AN in età adolescenziale impedisce il raggiungimento di un adeguato picco di massa ossea. La gravità dell'osteopenia è condizionata dalla durata della malattia, dall'età di insorgenza dell'amenorrea e dalla sua durata, dalla massa ossea al momento dell'esordio della malattia, da fattori genetici e dall'entità dell'attività fisica svolta. Il meccanismo della perdita ossea è multifattoriale: vi contribuiscono la denutrizione (in particolare l'insufficiente apporto di calcio, che aumenta il riassorbimento osseo), la scarsa produzione di IGF-I, l'ipercortisolismo, la carenza estrogenica e verosimilmente quella androgenica, in particolare quella del deidroepiandrosterone (DHEA). Sul versante terapeutico, sono stati sinora valutati gli effetti della somministrazione di estroprogestinici, DHEA, testosterone, IGF-I ricombinante e bisfosfonati in 
pazienti con AN. La somministrazione prolungata dell'associazione etinilestradiolo-levonorgestrel è apparsa in grado di ridurre i livelli circolanti degli indici di riassorbimento osseo e incrementare significativamente la densità minerale ossea (bone mineral density, BMD) a livello femorale, ma non a livello lombare. Il trattamento cronico con DHEA ha sortito gli stessi risultati della terapia estroprogestinica, ossia riduzione dei markers di riassorbimento osseo, incremento sovrapponibile della BMD femorale e nessun effetto sulla BMD lombare; inoltre, il DHEA ha determinato un aumento degli indici bioumorali di neoformazione ossea ed un miglioramento del quadro psicologico. La somministrazione sottocutanea per alcuni mesi di IGF-I ricombinante ha determinato un significativo aumento della BMD vertebrale in una vasta casistica di anoressiche osteopeniche, risultato non raggiunto con il solo estroprogestinico o con il DHEA; di grande interesse il fatto che il maggiore incremento della BMD lombare sia stato ottenuto con la somministrazione combinata di IGF-I biosintetico ed estroprogestinico. La somministrazione per nove mesi di risedronato ad un gruppo di pazienti anoressiche osteopeniche ha determinato una riduzione di circa il 30\% degli indici bioumorali di riassorbimento osseo ed un incremento di circa il 5\% della BMD lombare. Oltre alla necessità di ulteriori studi sulla sicurezza dell'uso prolungato di bisfosfonati nella giovane donna, va ricordata l'importanza, nell'AN, della supplementazione con calcio e metaboliti attivi del colecalciferolo.

\section{RIFERIMENTI BIBLIOGRAFICI}

1. Mehler PS, Brown C, Anorexia nervosa - medical complications, Journal of Eating Disorders, 2015: 3: 11.

2. Mehler PS, Rylander M, Bulimia nervosa - medical complications, Journal of Eating Disorders, 2015: 3: 12.

3. Misra M, Klibanski A, Endocrine consequences of anorexia nervosa, The Lancet Diabetes E Endocrinology, 2014: 2: 581-592.

4. Scacchi M, Pincelli AI, Caumo A, Tomasi P, Delitala G, Baldi G, Cavagnini F, Spontaneous nocturnal growth hormone secretion in anorexia nervosa, Journal of Clinical Endocrinology and Metabolism, 1997: 82: 3225-3229.

5. Scacchi M, Pincelli AI, Cavagnini F, Nutritional status in the neuroendocrine control of growth hormone secretion: the model of anorexia nervosa, Frontiers in Neuroendocrinology, 2003: 24: 200-224.

6. Misra M, Klibanski A, Anorexia nervosa and bone, Journal of Endocrinology, 2014: 221: R163-R176. 
